SLAC-PUB-11231

May 2005

\title{
SINGLE-BUNCH TUNE AND BEAM SIZE MEASUREMENTS ALONG BUNCH TRAINS IN PEP-II
}

\author{
Robert Holtzapple, ${ }^{\dagger}$ Alfred University, Alfred, NY 14802, USA \\ Denis Dujmic and Alan S. Fisher \\ Stanford Linear Accelerator Center, Stanford University, Stanford, CA 94305, USA
}

\begin{abstract}
By scanning gated cameras and gated tune monitors across the bunch pattern during normal colliding-bunch operation of PEP-II, the tunes and beam sizes of individual bunches were measured simultaneously in the high and low energy storage rings of PEP-II. The measurements were made with 1561 colliding bunches in PEP-II, arranged in trains of 66 bunches, with each bunch in the train separated by 4.2 ns. The tune and beam size measurements were correlated with the current, luminosity, and specific luminosity of the bunch. The results show a vertical tune shift at the start and end of the mini-trains, a luminosity droop along the mini-train, and specific luminosity drop in the first and last bunches of the train, since they experience a different parasitic crossing on either side of the interaction point (IP).
\end{abstract}

Presented at the IEEE Particle Accelerator Conference (PAC05), Knoxville, TN, 16-20 May 2005.

\footnotetext{
${ }^{*}$ Supported by U.S. Department of Energy contract DE-AC02-76SF00515.

${ }^{\dagger}$ Holtzapple@Alfred.edu
} 


\section{SINGLE-BUNCH TUNE AND BEAM SIZE MEASUREMENTS ALONG BUNCH TRAINS IN PEP-II}

\section{PEP-II OPERATING CONDITIONS}

PEP-II consists of two storage rings, a high-energy ring (HER) with 9-GeV electrons, and a low-energy ring (LER) with $3.1-\mathrm{GeV}$ positrons. The highest luminosity, $9.2 \times 10^{33} \mathrm{~cm}^{-2} \mathrm{sec}^{-1}$, was obtained by colliding 1588 bunches of 2.45A in the LER with 1.55A in the HER.

This high luminosity requires filling every second RF bucket, resulting in a small transverse separation-called a parasitic crossing - between bunches both entering and exiting the IP. The parasitic kicks the passing bunches give each other can shift the tune and lower the beam lifetime. If the beam is Gaussian transversely, the parasitic tune shift is given by [1]

$$
\left(\xi_{x}, \xi_{y}\right)=\frac{N r_{e}\left(\beta_{x},-\beta_{y}\right)}{2 \pi \gamma} \frac{\left(x^{2}-y^{2}\right)}{\left(x^{2}+y^{2}\right)^{2}}
$$

where $\gamma$ is the relativistic factor, $N$ is the number of particles, $x$ and $y$ are the horizontal and vertical beam separations, $\beta_{x}$ and $\beta_{y}$ are the horizontal and vertical betatron functions, and $r_{e}$ is the classical electron radius. Simulations of the PEP-II parasitic tune shift have been made, but no simple criteria determine an adequate beam separation [2]. Measurements presented here quantify some parameters affected by the parasitic crossing.

\section{EXPERIMENTAL APPARATUS}

\section{Gated Tune Tracker}

PEP's tune signals [3] are picked up at beam position monitor buttons. Horizontal and vertical difference signals are downconverted to baseband and displayed on a spectrum analyzer. Beam excitation can be added to the preamplifier input of bunch-by-bunch transverse feedback.

In 2002 we built horizontal and vertical tune-tracking loops for each ring [4]. When a sinusoidal excitation is swept in frequency across a tune peak, the phase of the response drops by $180^{\circ}$ relative to the drive. The linear slope at the center of the phase transition allows simple tracking without the dithering needed to follow an amplitude peak. The excitation frequency is adjusted to maintain the phase at the center of the transition, and this frequency, normalized to the revolution frequency, gives the fractional tune. A digital lock-in amplifier (Stanford Research Systems 830), which includes a sine source, measures both the amplitude and phase of the response at the drive frequency. A computer reads the phase and adjusts the frequency, using the slope of a phasefrequency curve obtained from an initial scan.

We pass the tune signal through a GaAs RF switch with a 2-ns rise, which we toggle once per turn. The excitation is similarly switched. By adding additional mixers, the signal from selected bunches can go to the tune trackers while signals from the rest go to the spectrum analyzer.
Using the gated tune tracker, $x$ and $y$-plane fractional tunes can be simultaneously measured in both rings.

\section{Gated Cameras}

PEP has two cameras (one per ring) with image intensifiers and 2-ns gates. These make it possible to measure the transverse size of individual bunches. Each camera images visible synchrotron light relayed from a dipole magnet to an optical table outside the radiation shielding. Each camera's trigger is synchronized with the revolution frequency of the ring and can be delayed to measure any bunch. Once a pair of colliding electron/positron bunches is selected, the horizontal and vertical projections of the images for both bunches can be fitted to Gaussians to determine the transverse width, peak, and mean of the images. We then can correlate the electron/positron beam sizes and tunes with other singlebunch parameters such as charge, luminosity, and specific luminosity.

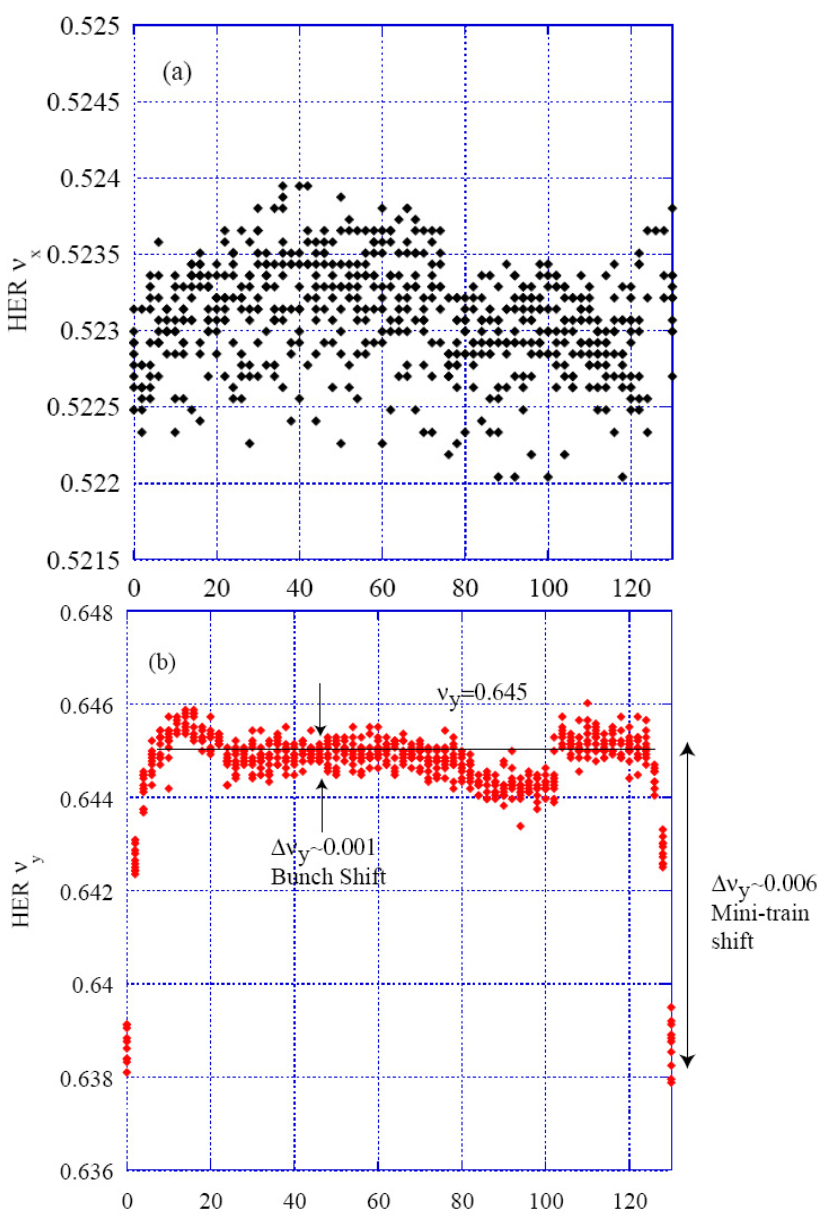

Figure 1. The (a) horizontal and (b) vertical tunes vs. RFbucket number (filling every $2^{\text {nd }}$ bucket) for a single train, measured for the first ten trains after the ion-clearing gap in the HER. 


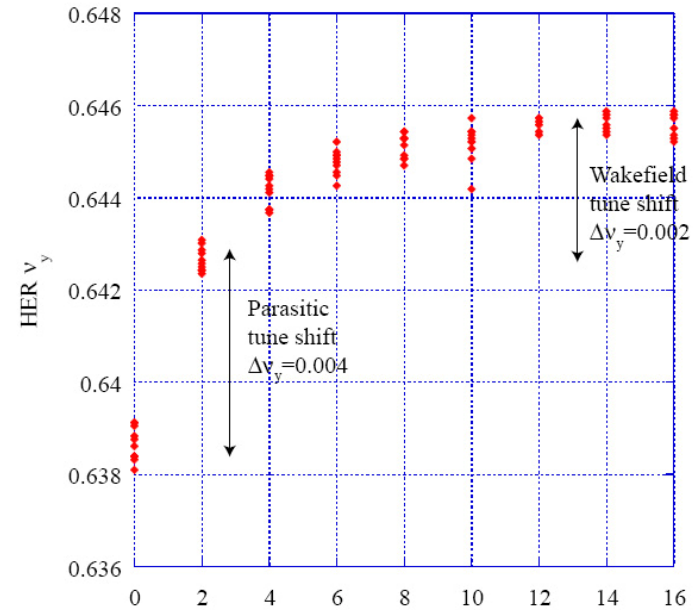

Figure 2. The vertical fractional tune for the HER at the beginning of the train.

\section{TUNES \& BEAM SIZE ALONG A TRAIN}

The following measurements were made parasitically while colliding 1561 bunches with average currents of $0.95 \mathrm{~mA} / \mathrm{bunch}$ of positrons and $1.46 \mathrm{~mA} / \mathrm{bunch}$ of electrons. The ring currents were constant due to trickle filling. Each train had 66 colliding bunches separated by 4.2 ns with a 5-bunch gap between trains. First and last bunches in a train experience only one parasitic crossing rather than two and will have a different tune shift.

The tunes and transverse size for colliding bunches were measured simultaneously in both rings for the first ten trains following the ion-clearing gap. Figs. 1 and 3 show the tunes for these trains for the HER and LER respectively.

For the HER, we observe: (1) a small peak-to-peak fractional horizontal tune variation along the trains, and (2) a strong vertical tune shift for the first and last two bunches of the train.

A detailed view of the HER vertical tune at the train's start (Fig. 2) and end (Fig. 1) shows: (1) a vertical parasitic tune shift of $\sim 0.004$ between the first two bunches, (2) a further shift of $\sim 0.002$ between bunches 2

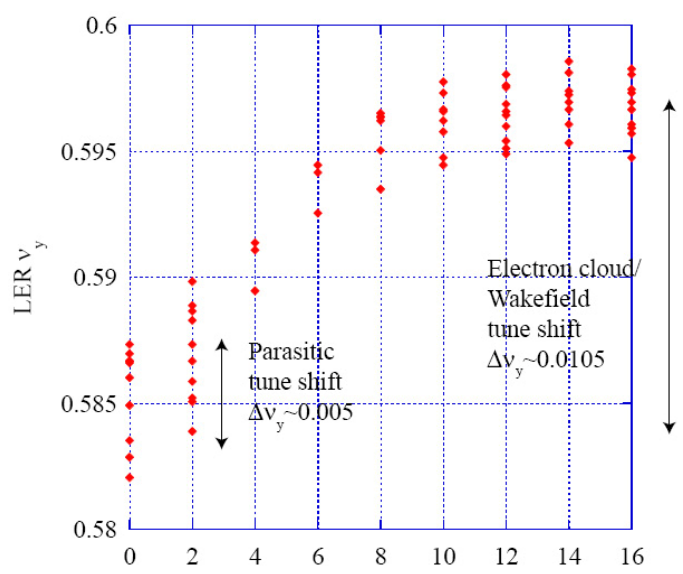

Figure 4. The vertical fractional tune for the LER at the beginning of the train.
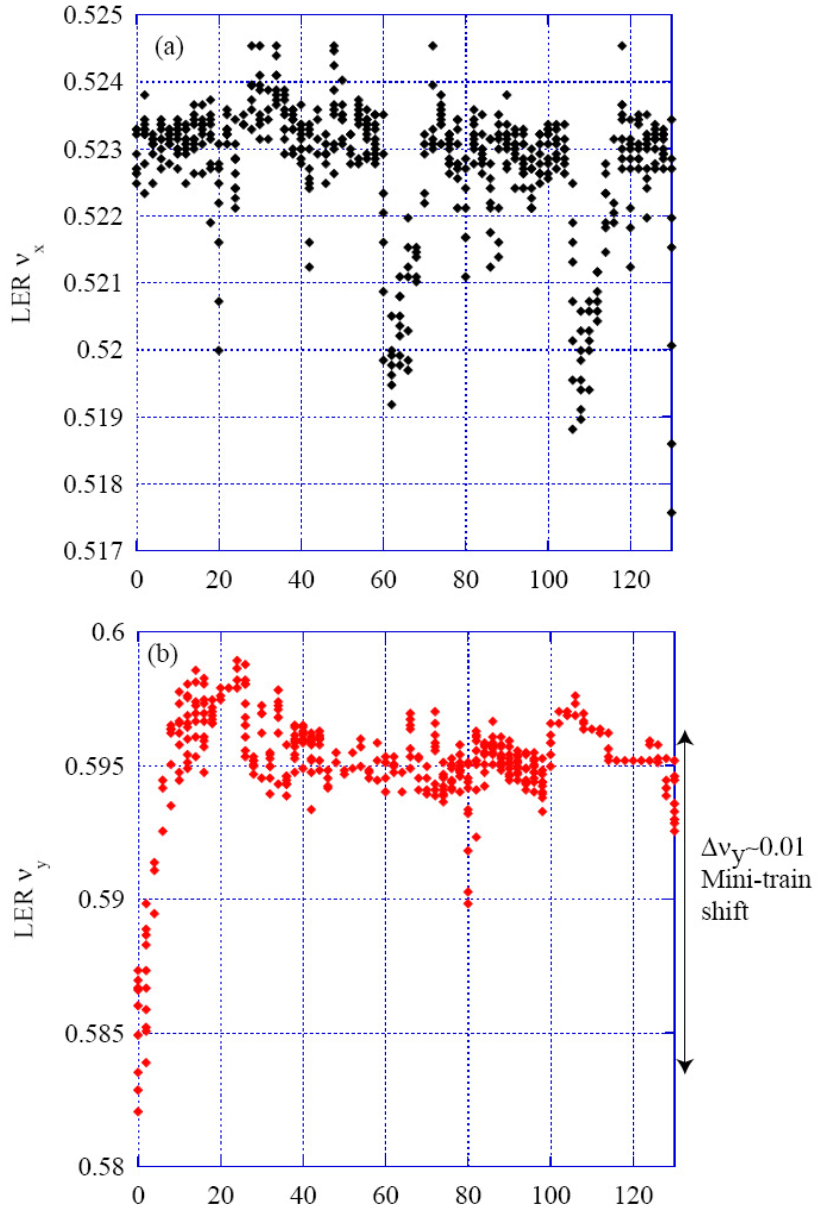

Figure 3. The (a) horizontal and (b) vertical tunes for a single train measured for the first 10 trains after the ion gap in the LER.

and 7, and (3) a vertical parasitic shift of $\sim 0.004$ between the final two.

In the LER (Fig. 3): (1) the horizontal tune varies along the train with a peak-to-peak variation of $\sim 0.005$ (presumably due to operator tuning), and (2) there is a strong vertical tune shift at the start of the train.

Like the HER, a detailed view of the LER vertical tune

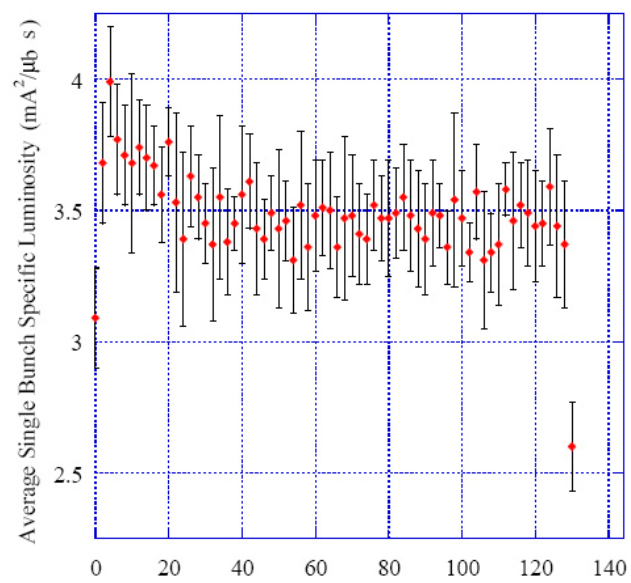

Figure 5. The average specific luminosity along the first ten trains. 


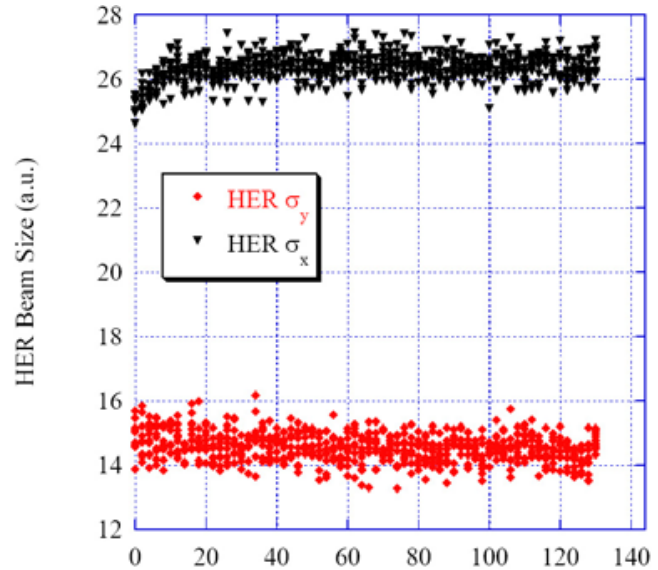

Figure 6. The HER vertical and horizontal beam size along the train.

at the beginning (Fig. 4) and end (Fig. 3) of the train shows: (1) a parasitic tune shift of $\sim 0.005$ between the first two bunches, (2) an additional shift between bunches 2 and 7, and (3) a small parasitic shift at the end.

The single-bunch specific luminosity and single-bunch beam sizes were also measured along the trains. The specific luminosity, shown in Fig. 5, peaks at bunch 3 and then drops down to a constant level around bunch 10 (bunch in the train 20). The first and last bunch in the trains has low luminosity due to the parasitic crossing.

The HER single-bunch beam size shows (Fig. 6): (1) a slight slope in $\sigma_{y}$ along the train and (2) a slightly smaller $\sigma_{x}$ at the start of each train.

For the LER, the single-bunch beam size shows (Fig. 7): (1) a fairly constant $\sigma_{y}$ along the trains and (2) a slightly larger $\sigma_{x}$ at the start of each train that appears to correlate with specific luminosity.

\section{SUMMARY}

In both rings, a strong vertical tune shift was measured at the start and end of the train. The shift appears to have two components: (i) a parasitic component at the train's start (between bunches 1 and 2) and end (bunches 65 to 66), and (ii) a gradual tune shift between bunches 2 and 7 . The gradual tune shift could be due to wakefields or, in the case of the LER, the electron cloud.

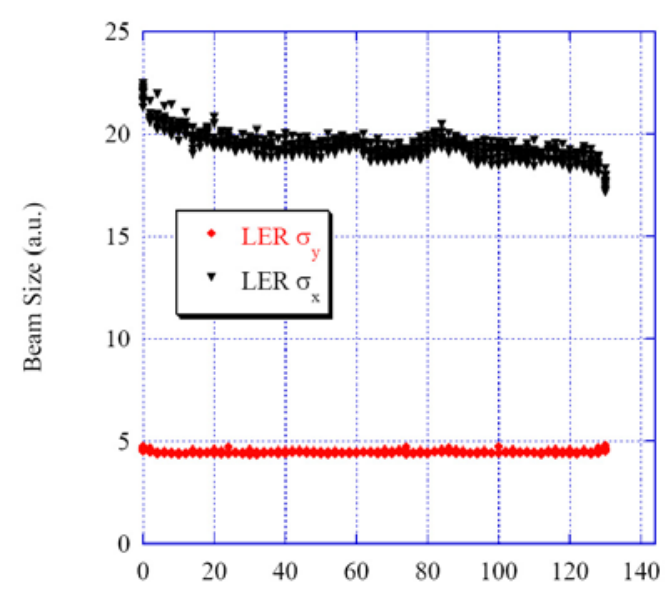

Figure 7. The LER vertical and horizontal beam size along the train.

The specific luminosity for the first and last bunch in the train is reduced due to the parasitic crossing. The luminosity peaks at the third bunch in the train and then drops down to a constant level around the tenth bunch.

The single bunch vertical and horizontal beam size in the LER and HER is fairly constant along the trains.

\section{REFERENCES}

[1] J. Jowett, "Beam-beam tune shift for Gaussian beams," Handbook of Accelerator Physics and Engineering, World Scientific.

[2] M.E. Biagini, "Long range beam-beam interaction in PEP-II," Oct. 2003. 30th Advanced ICFA Beam Dynamics Workshop on High Luminosity e+eCollisions (e+e- Factories 2003), Stanford, CA, 13-16 Oct 2003.

[3] A.S. Fisher, M. Petree, U. Wienands, S. Allison, M. Laznovsky, M. Seeman, and J. Robin, "Upgrades to PEP-II Tune Measurements," $10^{\text {th }}$ Beam Instrum. Workshop, Upton NY, 6-9 May 2002, AIP Conf. Proc. 648. Melville, NY: Amer. Inst. Phys, 2002, pp. 267-274.

[4] A.S. Fisher, M. Laznovsky, S.A. Allison, R.P. Chestnut, M. Petree, "Tune Feedback in PEP-II," IEEE NSS Conf., Portland, OR, Oct. 2003, SLACPUB-10230. 